\title{
Typologie des exploitations agropastorales du terroir de la NEMA (Sénégal, West Africa)
}

\author{
I. COLY ${ }^{1 *}$, F. DIOME ${ }^{3}$, H. DACOSTA ${ }^{2}$, R. MALOU ${ }^{4}$ et L. E. AKPO ${ }^{5}$ \\ ${ }^{1}$ MESUCURRS, Direction de la Recherche en Biotechnologies, BP 36005 Dakar, Sénégal. \\ ${ }^{2}$ FLSH/UCAD, Département de Géographie, BP 5005 Sénégal. \\ ${ }^{3}$ FST/UCAD, Institut des Sciences de la terre, BP 5005 Sénégal. \\ ${ }^{4}$ F ST/UCAD, Département de Géologie, BP 5 005, Sénégal. \\ ${ }^{5}$ F S T/UCAD, Laboratoire d'Ecologie \& d'Ecohydrologie, BP 5 005, Sénégal. \\ *Auteur correspondant, E-mail : i.coly@yahoo.fr
}

\section{RESUME}

Dans le terroir de la Néma, pour améliorer les rendements dans un contexte de baisse drastique des superficies cultivables et de la fertilité des sols, les agro-pasteurs ont recours à une gestion intégrée des productions agricoles et animales. Ainsi, suivant leur structure, les exploitations agricoles présentent des formes d'organisation diverses. Pour appréhender cette diversité, deux approches ont été utilisées : l'approche socio-économique (enquête) pour définir les caractéristiques des exploitations et l'approche agronomique visant à évaluer les ressources agricoles. Le traitement des données a permis de distinguer suivant la production et l'utilisation des résidus de récolte, deux groupes d'exploitations : celles qui produisent et utilisent, outre les autres résidus, les résidus de sorgho (A) et celles qui ne produisent et n'utilisent pas ces derniers (B); et suivant la taille, trois groupes d'exploitations : celles de grande taille (B1), de taille moyenne (A et B2) et de petite taille (B3). Si la production et l'épandage du fumier sont généralisés dans les groupes d'exploitations A, B1 et B2, ils sont peu développés dans les petites exploitations (B3). Et ce, du fait de l'absence ou de l'insuffisance $\mathrm{du}$ cheptel et de l'outillage agricole dans ces dernières, mais aussi de la faiblesse de la main d'œuvre. La main d'œuvre suivant les groupes d'exploitations est évaluée à 3,4 actifs agricoles dans le groupe B3 contre 6,$4 ; 7,8$ et 17 actifs agricoles respectivement pour les groupes d'exploitations B2, A et B1.

(C) 2011 International Formulae Group. All rights reserved.

Mots clés : Résidus de récolte, gestion intégrée, fertilité, fumier, unités agricoles.

\section{INTRODUCTION}

En milieu rural, le rapide accroissement démographique, avec comme corollaire l'accroissement des besoins des populations en produits alimentaires, a eu pour conséquence une très forte pression sur le milieu (Kaasschieter et Coulibaly, 1995 ; Bacco et al., 2003; Hauhouot, 2004).
L'augmentation des superficies emblavées semble ainsi quasi impossible. Il s'en est suivi une exploitation continue des sols sous culture et une diminution considérable $\mathrm{du}$ disponible fourrager car même les zones marginales, autrefois réservées aux animaux ont désormais été intégrées dans les systèmes de culture sous pluie. 
Les nouvelles terres cultivables, de même que les jachères sont devenues de plus en plus rares.

Dans le terroir de la Néma, depuis plusieurs années, les nouvelles terres à mettre en valeur sont devenues rares (Ngom, 2000), cela a entraîné la disparition de la pratique de jachère (Ngom, 2000 ; NDAO, 2001 ; Akpo et al., 2002, 2003). Dans ces conditions, ces terres continuellement cultivées ont commencé alors à montrer des problèmes de fertilité qui ont conduit à la baisse de leur potentiel productif (Razafindrakoto, 2007). Dès lors, la recherche de nouvelles stratégies d'exploitation des ressources, particulièrement en sols et fourrage, paraît intéressante pour alléger les pressions sur le milieu.

Ainsi pour satisfaire les besoins des populations et de leur cheptel, les paysans ont opté pour une intensification de l'agriculture. Celle-ci a consisté soit à une utilisation des engrais minéraux, essentiellement pour les cultures de rente (arachide), soit à une gestion intégrée des productions végétales et animales. L'association agriculture - élevage cherche à améliorer la production agricole sur de petites surfaces exploitées tout en apportant du fumier de ferme pour améliorer la fertilité du sol. Ce type de transfert est assuré par des flux de matières organiques et minérales générés par les déplacements des troupeaux d'une part, par le transport de ces matières par les populations vers les champs d'autre part.

Avec cette option de gestion intégrée des productions agricoles et animales, les résidus de récolte peuvent servir d'une part à la production de fourrage pour le bétail et d'autre part à la production de fumier et de compost. Ce type de fumure est utilisé pour améliorer la fertilité des champs. Le mode de gestion des résidus de récolte et de la fumure organique ainsi générée a donc une influence directe sur le maintien de la fertilité des sols.

Dans ce travail, nous cherchons, sur la base du mode de gestion des résidus de récolte et de la fumure organique mais aussi des caractéristiques sociodémographiques, à identifier et à décrire les différents types d'unités agropastorales.

\section{MATERIEL ET METHODES \\ Le terroir de la Néma \\ Le milieu physique}

Long de $11 \mathrm{~km}$ et large de $4,5 \mathrm{~km}$, le bassin versant de la Néma, couvre une superficie de $50 \mathrm{~km}^{2}$ (Lienou, 1995). Situé entre les latitudes $13^{\circ} 42^{\prime}$ et $13^{\circ} 45^{\prime}$ Nord et entre les longitudes $16^{\circ} 22^{\prime}$ 'et $16^{\circ} 29^{\prime}$ Ouest (Figure 1), dans la région naturelle du Sine, le terroir est limité au Sud et à l'Est par le bassin versant du Madina Djikoye, au Nord- Est par le bassin versant du fleuve Gambie, au NordOuest par les vallées côtières sous influence maritime. Il est drainé par une rivière de même nom, la Néma, orientée d'Est en Ouest, qui se jette dans le Bandiala, un bras de mer des îles du Saloum.

Sur le plan administratif, ce terroir appartient à la Région de Fatick, Département de Foundiougne. Des profils pédologiques (Figure 2) réalisés dans différentes parties du terroir (Coly, 1999) ont révélé 3 à 5 horizons.

L'horizon supérieur est partout sombre, mais plus noir dans la forêt-galerie et la zone de parcours que dans les champs. Sa texture est sableuse et sa structure en agrégats. Cependant dans la forêt-galerie, la texture est parfois argilo-sableuse. Les horizons sousjacents au supérieur ont une texture sableuse fine et une structure continue massive et leur couleur souvent beige. Il y apparaît très souvent des taches ocres (concrétions de fer).

La végétation, caractéristique des zones soudaniennes, a subi de profondes modifications dues à l'action anthropique et à la sécheresse. Le recouvrement est encore important dans la forêt-galerie $(70 \%)$, dans la zone de parcours (30\%), faible (5 à 10\%) dans les champs (Coly et al., 2001).

La forêt galerie, assez dense en aval et discontinue voire absente vers l'amont, borde le cours d'eau. On y rencontre en effet par endroits des champs, des parcelles de jachère et d'arbres fruitiers, qui sont des indices de transformation (Akpo et al., 2003).

\section{Les hommes et leurs activités}

La population du terroir de la Néma est de 4651 habitants répartis essentiellement 
entre 4 ethnies : Sérère (66\%), Wolof (18,5\%), Peuhl (7,4\%) et Mandingue (3,7\%).

L'agriculture, qui occupe plus de $75 \%$ de la population, concerne la production de l'arachide, du mil et du maïs en association ou en culture pure. L'arachide est en rotation avec le mil, rarement avec le maïs, dans un cycle continu arachide-mil-arachide; la jachère a quasiment disparu (Ngom, 2000).

La riziculture occupe principalement les femmes; elle est de moins en moins pratiquée en raison des problèmes d'eau (Mendy, 2000). L'arboriculture est la nouvelle forme d'occupation de l'espace; elle est représentée par des vergers installés dans les champs et la galerie forestière (Coly, 1999).

L'élevage est traditionnel, de type extensif. Les animaux sont nourris dans l'espace située entre les champs en saison des pluies et avec les fanes d'arachide en saison sèche (Ngom, 2000).

\section{Les méthodes utilisées}

Deux approches ont été développées : une approche socio-économique en vue de définir les caractéristiques des exploitations et une approche agronomique visant à évaluer les ressources agricoles. La première a reposé sur deux types d'enquêtes: une enquête de base et un suivi des exploitations. A partir des données obtenues à la suite de l'enquête de base, nous avons défini des types d'exploitations, en constituant des groupes pour celles qui présentent des caractéristiques similaires. La deuxième a consisté à évaluer la biomasse des résidus de récolte et les rendements des récoltes. Les productions et les utilisations des résidus de récolte ont été ainsi suivies dans des exploitations choisies dans les groupes précédents. Les résultats des deux approches nous ont permis de caractériser les groupes d'exploitations définis.

\section{Le choix des exploitations}

Une enquête destinée à analyser les éléments du système de production a été menée dans les 11 villages du terroir.
L'échantillon est constitué de 135 exploitations, soit un taux de sondage de $29,5 \%$. Elles ont été réparties au prorata du nombre d'exploitations par type de parc et par village (Tableau 1a).

Dans le terroir, 457 unités de production agricoles ont été recensées. Cellesci sont réparties dans les trois types de parcs (Figure 3) précédemment définis par Coly et al. (2005). Ce sont :

- le parc à Cordyla pinnata pour les champs de l'amont, ou parc à Dimb ;

- le parc à Parkia biglobosa pour les champs de plateaux, ou parc à Néré ;

- le parc à Pterocarpus erinaceus et Ficus sycomorus pour les champs de versants, ou parc mixte.

\section{L'enquête et le suivi des exploitations}

L'unité d'enquête et d'observation est le carré, qui caractérise l'unité de production. C'est une structure relativement facile à repérer pendant les enquêtes. C'est un groupe social caractérisé par un habitat commun (même concession) et qui est constitué d'un homme marié (chef de carré), de son (ses) épouse (s), de leurs enfants et d'éventuels dépendants mariés ou non. Le chef de carré correspond au chef d'exploitation.

L'enquête a consisté en des entretiens avec les chefs d'exploitations. Le questionnaire est de type fermé et ouvert. La fiche d'enquête comporte trois rubriques qui renseignent sur :

- les caractéristiques socio-démographiques de l'exploitation considérée ;

- la production, le ramassage et la gestion des résidus de récolte ;

- la fertilisation des champs (type d'engrais, quantité, ...).

Dans les différents groupes définis, des exploitations ont été retenues pour la suite des travaux.

Une seconde fiche d'enquête a alors été établie. Elle présente les rubriques suivantes : - identité de l'exploitation (nom du chef, âge, ethnie, personnes à charge, actifs, matériel) ; 
- superficies emblavées, cultures (rendement, production, résidus de récolte) ;

- facteurs de production (outillage disponible, animaux disponibles);

- revenu agricole des exploitations ;

- consommation des produits agricoles dans l'exploitation ;

- production et utilisation de la fumure animale dans l'exploitation ;

- effet du type d'engrais utilisé sur les rendements.

La quantité de fane d'arachide produite et ramassée est évaluée en nombre de charretées. Le nombre de sacs de fane par charretée a été ensuite déterminé.

Pour évaluer la charge moyenne d'une charrette de fumier répandu dans les champs, nous avons pesé la charge de 3 charrettes bovines.

\section{L'évaluation agronomique des exploitations}

Pour ce travail, nous avons utilisé la méthode des carrés de rendement, qui consiste à déterminer des placettes de $2 \mathrm{~m}$ de côté, et à récolter tous les plants d'arachide qui s'y trouvent. Nous avons ensuite séparé les gousses des fanes. Au total 20 carrés ont été utilisés par champ. Des échantillons de fanes et de gousses sont prélevés, pesés et mis en sachets et ramenés au laboratoire. Ils sont ensuite mis à sécher à l'étuve à $60^{\circ} \mathrm{C}$ jusqu'à poids constant.

\section{Traitement des données}

Afin de dégager les similitudes entre les exploitations du terroir sur le plan agropastoral et socio-économique et d'en déduire des groupes homogènes, la matrice 135 exploitations x 9 variables (avec 43 modalités) a été soumise à l'analyse factorielle des correspondances (AFC). L'analyse permet d'établir une correspondance entre ces deux ensembles.

Cette méthode a pour but de décrire sous forme de graphique le maximum de l'information contenu dans le tableau de données (exploitations et variables) appelé matrice. Ces deux ensembles de données sont disposés l'un en ligne (exploitations) et l'autre en colonne (variables).

L'interprétation des résultats repose sur l'inertie du système, qui indique le pouvoir explicatif d'un axe factoriel, et les contributions absolues et relatives, qui donnent respectivement l'importance des relevés (exploitations) et des variables dans la détermination des axes et la qualité de la représentation sur ceux-ci.

Les informations recueillies ont été codifiées et saisies sur le tableur Excel. Le tableau ainsi constitué est soumis à l'AFC sous le logiciel ADE4.

\section{RESULTATS}

\section{Caractéristiques démographiques des exploitations}

L'âge moyen des chefs d'exploitation dans le terroir de la Néma varie de 46 à 49 ans suivant les parcs et les villages (Tableau 2).

Les plus jeunes chefs d'exploitation sont rencontrés à Médina Santhie (35 ans), à Dielmo (40 ans), à Néma Bâ (42 ans) et à Néma Nding (45 ans). Les plus âgés sont de Keur Layène Socé (53), Santamba et Touba Nding (54 ans). Il y a une forte variation selon les villages.

L'âge moyen des chefs d'exploitation varie aussi en fonction des types de parcs : 46 ans dans les parcs à dimb, 49 ans dans le parc à néré et 48 ans dans le parc mixte.

Dans le terroir de la Néma, la taille moyenne des exploitations est variable. Le nombre moyen de personnes par exploitation varie de 8 dans le parc à néré, 10 dans le parc mixte, à 12 dans le parc à dimb. Il varie aussi selon le village considéré : de 6 à 17. Le village de Keur Oumar Mbombé renferme le nombre de personnes le plus élevé (17) alors que Sabouya présente le plus faible effectif (6).

Le nombre moyen d'actifs agricoles correspondant à la main d'œuvre est de 6,7 pour l'ensemble du terroir. La main d'œuvre varie de 5,4 actifs dans le parc à néré ; 7,5 actifs dans le parc mixte et de 9 actifs dans le parc à dimb. Elle varie aussi selon les villages, 
de 4,2 à Médina Santhie à 15,4 à Keur Oumar Mbombé. La main d'œuvre est relativement importante dans le parc à $\operatorname{dimb}$, particulièrement dans le village dit Keur Oumar Mbombé.

\section{Définition de l'hétérogénéité des exploitations}

Pour définir les groupes éventuels d'exploitations, l'analyse factorielle de correspondance a été appliquée à la matrice 135 exploitations x 9 variables.

L'inertie totale des points ainsi que la part de variance expliquée par chacun des 4 premiers axes factoriels sont portées dans le Tableau 3.

L'information contenue dans le tableau de données est de 1,67 bits dont 19\% sont projetées sur le plan des axes $\mathrm{F} 1$ et $\mathrm{F} 2$ et $31 \%$ pour l'ensemble des 4 premiers axes. On constate que l'information contenue dans le plan principal est élevée si on prend en compte le nombre important de variables. Ainsi le plan des axes F1 et F2 devra permettre alors une représentation simple et en deux dimensions de la structure majeure des données.

L'examen du Tableau 3 permet enfin de se rendre compte que l'information se stabilise autour du $3^{\text {ème }}$ axe (F3); il n'apparaît pas de différence importante entre les valeurs propres des axes factoriels F2 et F3.

Les contributions factorielles moyennes sont de 2,3 et de $0,7 \%$ pour les variables et les exploitations respectivement. Ainsi peuvent être retenus les points dont les contributions sont supérieures à la moyenne.

En ce qui concerne les variables, on retiendra la paille de sorgho en brûlis (RTSG) : 21,2\%, la culture du sorgho (SRG) : $18,9 \%$, l'utilisation de la paille de sorgho dans la confection des clôtures autour des habitations (CLSG) :17,7\%, le ramassage de la paille de sorgho (RSG) comme résidus de récolte: $16,6 \%$ et le village Néma Bâ (NMB) : 9,5\% pour l'axe F1. Ces valeurs contribuent à l'axe $\mathrm{F} 1$ pour $83,9 \%$. Elles indiquent ainsi clairement que l'axe F1 est surtout décrit par le village de Nema Bâ qui cultive le sorgho et dont les habitants ramassent la paille de sorgho pour la confection des clôtures. Le reste de la paille est brûlé dans les champs pour relever la fertilité des sols. L'axe F1 oppose ainsi dans les abscisses positives les exploitations dans lesquelles les résidus du sorgho sont utilisés (groupe A) de celles qui n'en font pas usage (groupe B) dans les abscisses négatives. Le village Nema Bâ paraît bien caractériser le premier groupe (Figure 4).

Les exploitations qui présentent de fortes contributions pour cet axe sont portées sur la Figure 3 ; il s'agit de 68(13,2\%), $66(11,8 \%), 61(10,2 \%), 69(9,8 \%), 67(7,9 \%)$, $41(5,2 \%), \quad 34(4,2 \%), \quad 63(3,9 \%), \quad 53(2,9 \%)$, $35(2,2 \%), 25(2 \%)$ et $23(1,6 \%)$.

Les variables déterminantes pour l'axe F2 sont représentées par la main d'œuvre (MNO1_2) : $16,4 \%$ et 5,3\%, Dielmo (DLM) : $12,3 \%$, l'embouche bovine (EMB) : 9,3\%, la présence des chevaux dans l'exploitation (EQN) : 7,4\%, Keur Oumar Mbombé (KOM) : 7,1\%, Ndoumboudj (NDB) : 6,3\%, l'utilisation de fourrages ligneux (EMO): $6,2 \%$ et Néma Nding (NMN): 5\%. La contribution de ces variables pour l'axe F2 est de $75,3 \%$. Ces variables définissent le groupe B qui correspond aux exploitations qui n'utilisent pas les résidus de sorgho. Dans ce groupe, un grand nombre d'exploitations présente des contributions supérieures à la moyenne.

Il n'y a pas d'exploitations qui présentent à la fois de fortes contributions pour les deux axes F1 et F2. Aussi, de nombreuses exploitations ont une contribution insignifiante.

Le long de l'axe F2, le groupe B peut être distingué en 3 sous-groupes. B1 situé du côté des ordonnées positives, est constitué d'exploitations qui pratiquent l'embouche bovine, l'émondage des ligneux et disposant des équins (animaux de trait). Ces exploitations sont particulièrement présentes dans les villages Keur Oumar Mbombé et Néma Nding. A l'opposé de l'axe, dans les ordonnées négatives, B3 correspond à des 
exploitations où la main d'œuvre est faible, l'élevage caprin est dominant. Ces exploitations concernent les villages de Dielmo, Ndoumboudj et Sabouya. Entre B1 et $\mathrm{B} 3$, le sous groupe $\mathrm{B} 2$, réparti à la fois dans les abscisses négatives et positives de l'axe, correspond à des exploitations dans lesquelles la main d'œuvre comprendrait 5 à 10 actifs agricoles. Ces exploitations seraient réparties partout dans le terroir. L'axe F2 définirait ainsi la taille des exploitations.

L'axe factoriel F2 distinguerait alors les grandes exploitations (B1), les exploitations de taille moyenne (B2) et les exploitations de petite taille ou petites exploitations (B3).

Par rapport à la position centrale des groupes A sur l'axe F2, ce groupe pourrait être considéré comme renfermant des exploitations moyennes. Ainsi Les groupes A et $\mathrm{B} 2$ définiraient le groupe des exploitations moyennes.

\section{Description des groupes d'exploitations}

Les valeurs moyennes des différents critères et indicateurs (variables) (Tableau 1b) ont été utilisés pour décrire les groupes d'exploitation.

\section{Caractéristiques socio-économiques}

Dans le Tableau 4 sont présentés les critères et indicateurs socio-économiques. L'âge du Chef d'exploitation où la paille de sorgho est utilisée est plus élevé (53 ans) que dans les autres groupes. Les petits exploitants ne produisent ni le sorgho, ni le melon, ni du maïs.

Tous les grands exploitants possèdent des animaux de transport (équins), pratiquent l'embouche et utilisent le fumier de ferme (par la stabulation des animaux à l'engrais).

Il y a beaucoup plus de personnes dans le GB1 que partout ailleurs. Il utilise aussi plus d'actifs agricoles.

Dans le Tableau 5, nous avons indiqué les paramètres agronomiques des différents groupes d'exploitations.
La visite des exploitations nous a permis de nous rendre compte que tous les fermiers produisent du fumier de ferme (même avec des petits ruminants en libre pâture). Ils disposent en effet des bœufs de trait ou des caprins et des ovins.

\section{Production de résidus de récolte}

Le Tableau 6 présente les résultats de suivi de production de fane obtenu par des mesures au champ pour les deux campagnes de production.

Les rendements et productions moyens de fanes obtenues à partir des données des deux approches (socio-économique et agronomique) sont présentés dans le Tableau 7.

\section{Synthèse : les types d'exploitations}

La typologie du bassin de la Néma a reposé ainsi sur deux facteurs, d'une part, la spéculation « sorgho » et l'utilisation de ses résidus et d'autre part la taille de l'exploitation agricole. Toutefois, sur le plan pratique, il apparaît une prépondérance du facteur taille de l'exploitation. Ce facteur représenté par l'axe F2 permet de distinguer globalement au niveau des ordonnées positives le groupe des exploitations de grande taille, qui s'oppose à celui des exploitations de petite taille situées au niveau des ordonnées négatives. Entre ces deux groupes, on a distingué un troisième groupe, des exploitations moyennes, constitué par les exploitations du groupe A et du sous groupe B2 (Figure 5).

\section{Les grandes exploitations}

Ces exploitations sont composées de 17 actifs agricoles et 19 personnes à charge. Les chefs d'exploitation sont âgés de 49 ans environ. Dans ce groupe, $40 \%$ des exploitations cultivent le melon et tous (100\%) pratiquent l'embouche bovine (Tableau 4). Les superficies emblavées sont de 5,75 ha pour l'arachide; 6,75 ha pour le mil et de 0,75 ha pour le maïs (Tableau 5). 
La production moyenne de fane par exploitation est de 9,15 tonnes (Tableau 7) dont la plus grande partie (environ 93\%) est destinée à l'alimentation animale. Le rendement moyen est de 1,59 t par ha. $60 \%$ des exploitants vendent la fane d'arachide. La quantité individuelle vendue est de 46 sacs en moyenne. C'est aussi dans ce groupe que l'épandage du fumier dans les champs est généralisé avec une quantité moyenne de 20 charrettes par an.

Le cheptel est composé de 8 bovins en élevage extensif, 2 bovins de trait, 1 asin, 2 équins, 2 ovins et 1 caprin. L'outillage agricole est constitué de 3 houes, 2 semoirs, 1 souleveuse, 2 charrettes et 4 dabas (Tableau 5). Ce sont des groupes que nous avons appelés des exploitations agropastorales.

\section{Les exploitations de taille moyenne}

C'est un groupe dont une partie des exploitations cultive le sorgho dont elles ramassent la paille pour les clôtures des habitations. Ces exploitations comportent 9 à 11 personnes à charge et 7 ou 8 actifs agricoles en moyenne. L'âge moyen des chefs d'exploitation varie de 46 à 53 ans. Une bonne partie des exploitations de ce groupe pratique la culture du melon.

Les superficies emblavées varient de 3,42 à 3,83 ha pour l'arachide ; 3,42 à 4,5 ha pour le mil et 0 à 0,2 ha pour le sorgho (Tableau 5).

Le rendement en fane d'arachide varie de $92,5 \mathrm{~kg} / \mathrm{ha}$ dans les exploitations ne pratiquant pas le spéculation du sorgho à $1490 \mathrm{~kg} / \mathrm{ha}$ dans celles pratiquant cette spéculation. La production moyenne à l'échelle des exploitations évaluée à 3,80 tonnes (Tableau 7) est presque entièrement destinée à l'alimentation du bétail (>90\%, soit $3678 \mathrm{~kg}$ ) dans les exploitations qui ne cultivent pas le sorgho. Elle est de 5,1 tonnes dans celles qui produisent et utilisent les résidus de sorgho.
Bien que la vente de la fane d'arachide soit pratiquée par 40,8\% des exploitants ne cultivant pas le sorgho, la quantité vendue est relativement très faible : 9 sacs en moyenne par exploitation, soit $126 \mathrm{~kg}$. Parmi les exploitations agricoles qui cultivent le sorgho (GA), 25\% vendent leur fane d'arachide à hauteur de 25 sacs par exploitation soit $10 \%$ de la production totale, le reste est destiné à l'alimentation du bétail.

L'utilisation du fumier est en revanche importante. Elle est pratiquée par $81 \%$ et $87 \%$ respectivement pour les exploitations pratiquant la culture du sorgho et celles ne la pratiquant. Chaque exploitant utilise en moyenne 7 charretées.

Le cheptel est constitué de 2 à 6 bovins en élevage extensif, de 3 à 4 bovins de trait, de 1 à 2 ovins, d'un asin et de 1 à 2 caprins. L'outillage agricole est formé de 2 à 3 houes, de 2 à 3 semoirs, d'une souleveuse, d'une charrette, de 2 hilaires et de 3 à 5 dabas par exploitation.

\section{Les petites exploitations}

Ce groupe est caractérisé par 5 personnes à charge et 4 actifs agricoles. L'âge des chefs d'exploitation est le plus faible : 42 ans. Le rendement moyen en fane d'arachide est de $1270 \mathrm{~kg}$. La fane produite, environ $2032 \mathrm{~kg}$ (Tableau 7) par exploitation, est presque totalement destinée à l'alimentation du bétail (1997 kg). C'est le groupe qui vend le moins la fane d'arachide: 18,2\% des exploitants avec seulement 4 sacs en moyenne. Ce groupe ne pratique pas l'embouche bovine. Seules les spéculations de l'arachide et du mil sont pratiquées dans ce groupe.

L'épandage du fumier n'est pas aussi assez développé; peu d'exploitants $(9,1 \%)$ l'utilisent. La quantité de fumier répandue par exploitant est aussi très faible : 0,36 charretée. C'est le groupe qui dispose le moins d'animaux et d'outillage agricole (Tableau 5). 
Tableau 1a : Répartition des fiches d'enquête selon les types de parcs et les villages du terroir de la Néma.

\begin{tabular}{|c|c|c|c|c|c|}
\hline \multirow[t]{2}{*}{ Parcs à } & \multirow[t]{2}{*}{ Villages } & \multicolumn{2}{|c|}{$\begin{array}{l}\text { Exploitations selon } \\
\text { le parc }\end{array}$} & \multicolumn{2}{|c|}{ Exploitations par village } \\
\hline & & Total & Echantillon & TOTAL & Echantillon \\
\hline & -Dielmo & & & 41 & 12 \\
\hline \multirow[t]{4}{*}{$\operatorname{Dimb}$} & -K. Layène Fatim & 124 & 37 & 67 & 20 \\
\hline & -K. Oumar Mbombé & & & 16 & 5 \\
\hline & -K. Layène Socé & & & 16 & 6 \\
\hline & -Médina Santhie & & & 19 & 5 \\
\hline \multirow[t]{4}{*}{ Néré } & -Santamba & 185 & 55 & 38 & 11 \\
\hline & -Sabouya & & & 15 & 4 \\
\hline & -Ndoumboudj & & & 97 & 29 \\
\hline & -Néma Bâ & & & 79 & 23 \\
\hline \multirow[t]{2}{*}{ Mixte } & -Néma Nding & 147 & 43 & 57 & 17 \\
\hline & -Touba Nding & & & 11 & 3 \\
\hline Total & 11 villages & 457 & 135 & 457 & 135 \\
\hline
\end{tabular}

Tableau 1b : Liste des principaux critères (9) et indicateurs (43) utilisés.

\begin{tabular}{|c|c|c|}
\hline Variables & Codes & Modalités \\
\hline \multirow[t]{11}{*}{ Villages } & TBN & Touba Nding \\
\hline & DLM & Dielmo \\
\hline & NMN & Néma Nding \\
\hline & STB & Santamba \\
\hline & SBY & Sabouya \\
\hline & MDS & Médina Santhie \\
\hline & NMB & Néma Ba \\
\hline & NDB & Ndoumbout \\
\hline & KLS & Keur L. Socé \\
\hline & KLF & Keur L. Fatim \\
\hline & KOM & Keur O. Mbombé \\
\hline \multirow[t]{3}{*}{ Main d'œuvre (actifs agricoles) } & MNO1 & Main d'oeuvre $(<5)$ \\
\hline & MNO2 & Main d'oeuvre (entre 5 et 10 ) \\
\hline & MNO3 & Main d'oeuvre (> 10) \\
\hline \multirow[t]{5}{*}{ Spéculations } & ARA & Arachide \\
\hline & MIL & Mil \\
\hline & MAS & Maïs \\
\hline & MEL & Melon \\
\hline & SRG & Sorgho \\
\hline \multirow[t]{4}{*}{ Résidus de récolte ramassés } & RAR & Fane d'arachide \\
\hline & RML & Paille de mil \\
\hline & RMS & Paille de maïs \\
\hline & RSG & Paille de Sorgho \\
\hline \multirow[t]{4}{*}{ Usages des résidus de récolte } & ALAR & Fane d'arachide pour l'alimentation du bétail \\
\hline & VTAR & Vente fane d'arachide \\
\hline & RTML & Brûlis paille de mil \\
\hline & \multicolumn{2}{|c|}{1948} \\
\hline
\end{tabular}




\begin{tabular}{lll} 
& RTMS & Brûlis paille de maïs \\
& RTSG & Brûlis paille de Sorgho \\
& CLML & Paille de mil dans les clôtures \\
& CLSG & Paille de Sorgho dans les clôtures \\
\hline Composition du cheptel & BOV & Bovins \\
& EQN & Equins \\
& ASN & Asins \\
& OVN & Ovins \\
& CAP & Caprins \\
\hline Systèmes d'élevage & EMB & Pratique de l'embouche bovine \\
& DIV & Elevage extensif \\
& STB & Pratique de la stabulation \\
& GAR & Gardiennage des animaux \\
\hline Ligneux fourrager & EMO & Emondage ligneux pour alimentation du bétail \\
\hline Usages du fumier & EPA & Epandage du fumier dans les champs \\
& CCA & Epandage du fumier dans les champs de case \\
& CBR & Epandage fumier dans les champs de brousse \\
\hline
\end{tabular}

Tableau 2 : Caractéristiques démographiques des exploitations (C.E. = Chef d'exploitation).

\begin{tabular}{lllll}
\hline & & & \multicolumn{2}{c}{ Effectif des personnes } \\
\hline Parc à & Villages & Age C.E (ans) & Total & Actifs \\
\hline \multirow{4}{*}{ Dimb } & Keur Layène Fatim & 49,3 & 9,7 & 6,9 \\
& Keur Oumar Mbombé & 48,6 & 17 & 15,4 \\
& Dielmo & 40,1 & 6,4 & 4,6 \\
& Moyenne & $\mathbf{4 6}$ & $\mathbf{1 1 , 1 8}$ & $\mathbf{9 , 0}$ \\
& Santamba & 54,1 & 8,7 & 5,7 \\
& Sabouya & 50,5 & 6,3 & 4,8 \\
& Ndoumboudj & 49,1 & 8,0 & 6,1 \\
& Médina Santhie & 35,4 & 8,2 & 4,2 \\
& Keur Layène Socé & 53,1 & 7,5 & 6,1 \\
& Moyenne & $\mathbf{4 8 , 5}$ & $\mathbf{7 , 7}$ & $\mathbf{5 , 4}$ \\
\multirow{4}{*}{ Mixte } & Néma Ba & 42,3 & 9,2 & 6,8 \\
& Néma Nding & 44,9 & 12,0 & 8,8 \\
& Touba Nding & 54,3 & 9,0 & 6,9 \\
& Moyenne & $\mathbf{4 7 , 2}$ & $\mathbf{1 0 , 1}$ & $\mathbf{7 , 5}$ \\
\hline
\end{tabular}

Tableau 3 : Valeurs propres (bits) et inertie (\%) des premiers axes de l'AFC.

\begin{tabular}{lllll}
\hline Axes & $\mathrm{F} 1$ & $\mathrm{~F} 2$ & $\mathrm{~F} 3$ & $\mathrm{~F} 4$ \\
\hline Valeurs propres & 0,19 & 0,13 & 0,12 & 0,1 \\
Inertie (\%) & 11,45 & 7,60 & 6,78 & 5,92 \\
Inerties cumulées & 11,45 & 19 & 25,83 & 31 \\
\hline
\end{tabular}


Tableau 4 : Caractéristiques des groupes d'exploitations du terroir de la Néma.

\begin{tabular}{|c|c|c|c|c|c|c|}
\hline \multirow[t]{2}{*}{ CRITERES } & \multirow[t]{2}{*}{ VARIABLES } & \multirow[t]{2}{*}{ CODES } & \multicolumn{4}{|c|}{ Groupes d'exploitations } \\
\hline & & & G.A & G.B1 & G.B2 & G.B3 \\
\hline \multirow{3}{*}{ Actifs agricoles } & Age (ans) & Age & 53 & 49 & 46 & 42 \\
\hline & Nombre moyen personnes & Nmp. & 11 & 19 & 9 & 5 \\
\hline & Main d'œuvre & MNO & 7,8 & 17 & 6,4 & 3,4 \\
\hline \multirow{5}{*}{ Spéculations } & Arachide $(\%)$ & AR. & 100 & 100 & 99 & 90,9 \\
\hline & $\operatorname{Mil}(\%)$ & MIL & 100 & 100 & 100 & 91 \\
\hline & Maïs (\%) & MAS & 38 & 80 & 36 & 0 \\
\hline & Melon (\%) & MEL & 0 & 40 & 3,9 & 0 \\
\hline & Sorgho $(\%)$ & SRG & 88 & 0 & 0 & 0 \\
\hline $\begin{array}{l}\text { Rendement } \\
\text { estimé }\end{array}$ & Fane d'arachide (charretées) & Far. (ch) & 3,1 & 3,4 & 3,9 & 1,95 \\
\hline \multirow[t]{5}{*}{ Ramassage } & Fane d'arachide (\%) & $\mathrm{AR}$ & 100 & 100 & 99 & 91 \\
\hline & Paille de mil (\%) & MIL & 63 & 100 & 84 & 36 \\
\hline & Paille de Sorgho (\%) & SRG & 38 & 0 & 0 & 0 \\
\hline & $\begin{array}{l}\text { Fane d'arachide pour alim. bét. } \\
(\%)\end{array}$ & F.AR_AL & 100 & 100 & 97,1 & 90,9 \\
\hline & Vente $(\%)$ & VTE & 25 & 60 & 40,8 & 18,2 \\
\hline \multirow{8}{*}{ Utilisations } & Rest. Paille de mil(\%) & RET_P.MIL & 100 & 80 & 98,1 & 72,7 \\
\hline & Rest. Paille de maïs(\%) & RET_P.MAS & 38 & 80 & 35,9 & 0 \\
\hline & Rest. Paille de sorgho(\%) & RET_P.SRG & 88 & 0 & 0 & 0 \\
\hline & Paille de mil pour clôture(\%) & CLO_P.MIL & 69 & 80 & 79,6 & 27 \\
\hline & $\begin{array}{l}\text { Paille de sorgho pour } \\
\text { clôture(\%) }\end{array}$ & CLO_SRG & 38 & 0 & 0 & 0 \\
\hline & Bovins (\%) & BOV & 88 & 100 & 86 & 36 \\
\hline & Equins (\%) & EQU & 6,3 & 100 & 21 & 36 \\
\hline & Asins $(\%)$ & ASI & 25 & 60 & 43 & 18 \\
\hline \multirow[t]{5}{*}{ Animaux } & Ovins $(\%)$ & $\mathrm{OV}$ & 6,3 & 20 & 24 & 9,1 \\
\hline & Caprins (\%) & CAP & 6,3 & 0 & 8,74 & 9,09 \\
\hline & Embouche $(\%)$ & EMB & 6,3 & 100 & 11 & 0 \\
\hline & Divagation (\%) & DIV & 31 & 60 & 50 & 27 \\
\hline & Stabulation (\%) & STAB & 100 & 100 & 96,1 & 45,5 \\
\hline \multirow{2}{*}{$\begin{array}{l}\text { Conduite du } \\
\text { bétail }\end{array}$} & Gardiennage (\%) & GARD & 38 & 40 & 22,3 & 36,4 \\
\hline & Emondage & EMO & 6,3 & 40 & 8,7 & 9,1 \\
\hline Fane & $\begin{array}{l}\text { Quantité de fane d'arachide } \\
\text { vendue (sacs) }\end{array}$ & QTE (sacs) & 16 & 54 & 15,3 & 7,27 \\
\hline \multirow[t]{2}{*}{ Fumier de ferme } & Epandage de fumier (\%) & OUI & 81 & 100 & 87 & 9,1 \\
\hline & Quantité de fumier charettes) & $\begin{array}{l}\text { QTE/an } \\
\text { (char) }\end{array}$ & 6,9 & 20 & 7 & 0,36 \\
\hline
\end{tabular}


Tableau 5 : Variation des paramètres agronomiques suivis selon les groupes d'exploitations.

\begin{tabular}{|c|c|c|c|c|c|}
\hline \multirow{2}{*}{$\overline{\text { Paramètres }}$} & & \multicolumn{4}{|c|}{ Groupes d'exploitations } \\
\hline & & G. $\mathbf{A}$ & G. B1 & G. B2 & G. B3 \\
\hline \multirow{4}{*}{ Superficies moyennes emblavées (ha) } & Arachide & 3,42 & 5,75 & 3,83 & 1,6 \\
\hline & Mil & 4,5 & 6,75 & 3,42 & 1,9 \\
\hline & Maïs & 0 & 0,75 & 0,2 & 0 \\
\hline & Sorgho & 0,2 & 0 & 0 & 0 \\
\hline \multirow{5}{*}{ Rendement moyen $(\mathrm{kg} / \mathrm{ha})$} & Gousses arachide & 530,14 & 892,76 & 202,4 & 419,83 \\
\hline & Fane d'arachide & 1314,63 & 1337,28 & 790,64 & 1412,66 \\
\hline & Mil & 114,81 & 505 & 367,99 & 270,84 \\
\hline & Maïs & 0 & 350 & 50 & 0 \\
\hline & Sorgho & 16,67 & 0 & 0 & 0 \\
\hline \multirow{5}{*}{ Production moyenne $(\mathrm{kg})$} & Gousses arachide & 1813 & 5130 & 775 & 6720 \\
\hline & Fane d'arachide & 4497 & 7689 & 3028 & 2260 \\
\hline & Mil & 741,67 & 3410 & 1260 & 550 \\
\hline & Maïs & 0 & 262 & 10 & 0 \\
\hline & Sorgho & 10 & 0 & 0 & 0 \\
\hline \multicolumn{2}{|c|}{ Quantité de fane d'arachide ramassée $(\mathrm{kg})$} & 5104,67 & 7659 & 3148,5 & 2246,48 \\
\hline \multicolumn{2}{|l|}{ Quantité de fane d'arachide vendue (sacs) } & 33,33 & 37,5 & 2,5 & 0 \\
\hline \multicolumn{2}{|c|}{ Quantité de fane d'arachide destinée à l'alimentation bétail (kg) } & 4644,6 & 7141,5 & 3114,27 & 2246,48 \\
\hline \multirow{6}{*}{ Outillage agricole } & Houe & 3 & 3 & 2 & 1 \\
\hline & Semoir & 3 & 2 & 2 & 1 \\
\hline & Souleveuse & 0 & 1 & 1 & 0 \\
\hline & Charrette & 1 & 2 & 1 & 1 \\
\hline & Hilaire & 2 & 0 & 2 & 1 \\
\hline & Daba & 5 & 4 & 3 & 1 \\
\hline \multirow{6}{*}{ Composition du cheptel } & Bovins extensifs & 6 & 8 & 2 & 1 \\
\hline & Bovins de trait & 3 & 2 & 4 & 2 \\
\hline & Asins & 1 & 1 & 1 & 1 \\
\hline & Equins & 0 & 2 & 0 & 0 \\
\hline & Ovins & 1 & 2 & 2 & 0 \\
\hline & Caprins & 2 & 1 & 1 & 1 \\
\hline \multirow{2}{*}{ Revenu agricole moyen (FCFA) } & Arachide & 175450 & 619120 & 38315 & 34285 \\
\hline & Mil & 0 & 90000 & 0 & 0 \\
\hline \multirow{2}{*}{ Consommation journalière $(\mathrm{kg})$} & Riz & 2,25 & 3,75 & 3,17 & 2 \\
\hline & Mil & 3,08 & 6 & 3,5 & 2,7 \\
\hline \multicolumn{2}{|l|}{ Quantité de fumier par 1 ha (kg) } & 461,4 & 1173,3 & 1100,0 & 158,4 \\
\hline
\end{tabular}

\section{DISCUSSION}

Ce travail a identifié les différents groupes d'exploitations agropastorales sur la base de leurs caractéristiques sociodémographiques et leur mode de gestion des résidus de récolte. Les données ayant servis à la caractérisation de ces groupes sont obtenues à partir des approches socioéconomique et agronomique.

\section{Les caractéristiques des exploitations}

Le terroir de la Néma est majoritairement peuplé par de Serères, dits Sérères niominca, qui sont des agro-pasteurs. Dans ce milieu, les exploitations sont des unités agro-pastorales 
Tableau 6 : Production de la fane d'arachide selon les groupes d'exploitations pour les campagnes 2000-2001 et 2001-2002.

\begin{tabular}{|c|c|c|c|c|c|c|c|c|}
\hline & \multicolumn{4}{|c|}{ 2000-2001 } & \multicolumn{4}{|c|}{ 2001-2002 } \\
\hline & GA & GB1 & GB2 & GB3 & GA & GB1 & GB2 & GB3 \\
\hline $\begin{array}{l}\text { Superf. Emblavée } \\
\text { /expl. (ha) }\end{array}$ & 3,41 & 5,75 & 3,83 & 1,6 & 3,42 & 5,75 & 3,83 & 1,6 \\
\hline $\begin{array}{l}\text { Effectif (nbre } \\
\text { d'expl.) }\end{array}$ & 54 & 17 & 352 & 34 & 54 & 17 & 352 & 34 \\
\hline Rendement (kg) & 1949 & 2642 & 1556,3 & 1493,6 & 1381,31 & 1051,8 & 832,31 & 761,5 \\
\hline $\begin{array}{l}\text { Prod. } \\
\text { moy./expl.(tonnes) }\end{array}$ & $\begin{array}{l}6,65 \\
9\end{array}$ & 15,19 & 5,966 & 2,239 & 4,719 & 6,048 & 3,190 & 1,218 \\
\hline $\begin{array}{l}\text { Production de fane } \\
\text { (tonnes) }\end{array}$ & $\begin{array}{l}359, \\
6\end{array}$ & 258,3 & 2099,9 & 81,253 & 254,85 & 102,81 & 1123,1 & 41,426 \\
\hline $\begin{array}{l}\text { Prod. moy./expl. } \\
\text { dans le terroir } \\
\text { (tonnes) }\end{array}$ & 7,5 & & & & 3,79 & & & \\
\hline
\end{tabular}

Tableau 7 : Valeurs moyennes de quelques paramètres évalués à partir des deux approches pour les différents groupes d'exploitations.

\begin{tabular}{lcccc}
\hline & Groupe A & Groupe B1 & Groupe B2 & Groupe B3 \\
\hline $\begin{array}{l}\text { Rendement moyen de fane } \\
(\mathrm{kg}) / \text { exploitation }\end{array}$ & 1490 & 1592 & 992 & 1270 \\
\hline $\begin{array}{l}\text { Production moy. } \\
\text { fane/exploitation (kg) }\end{array}$ & 5080 & 9154,0 & 3801 & 2032 \\
\hline $\begin{array}{l}\text { Quantité moy. fane vendue } \\
\text { (sacs)/exploitation }\end{array}$ & 25 & 46 & 9 & 4 \\
\hline $\begin{array}{l}\text { Quantité moy. de fane } \\
\text { destinée à l'alimentation du } \\
\text { bétail (kg)/ exploitation }\end{array}$ & 4738 & 8524 & 3678 & 1977 \\
\hline
\end{tabular}

qui associent agriculture (productions végétales) et élevage (dont les animaux sont utilisés pour les travaux agricoles).

Deux grands groupes d'exploitations ont été définis; ce sont les exploitations du groupe A, qui sont caractérisées par la culture du sorgho, et celles du groupe B qui n'en font pas. Dans le dernier, nous avons aussi identifié suivant la taille de grandes (B1) de moyennes (B2) et de petites (B3) exploitations. Le groupe B3 n'utilise pas l'engrais chimique à cause certainement de leur moyen financier limité.

\section{La disponibilité des sous-produits agricoles}

Les résultats obtenus montrent que les facteurs déterminants de la production de fane sont la taille des exploitations et le nombre d'exploitations par groupe. En effet les exploitations moyennes représentent plus de $75 \%$ de l'effectif total, les grandes exploitations sont inférieures à 5\%, les petites exploitations représentent $8 \%$. La production des grandes exploitations est supérieure à celle des petites à cause de leur taille. La production apparaît donc corrélée à la taille de l'exploitation d'une part et à l'effectif du groupe d'autre part. 


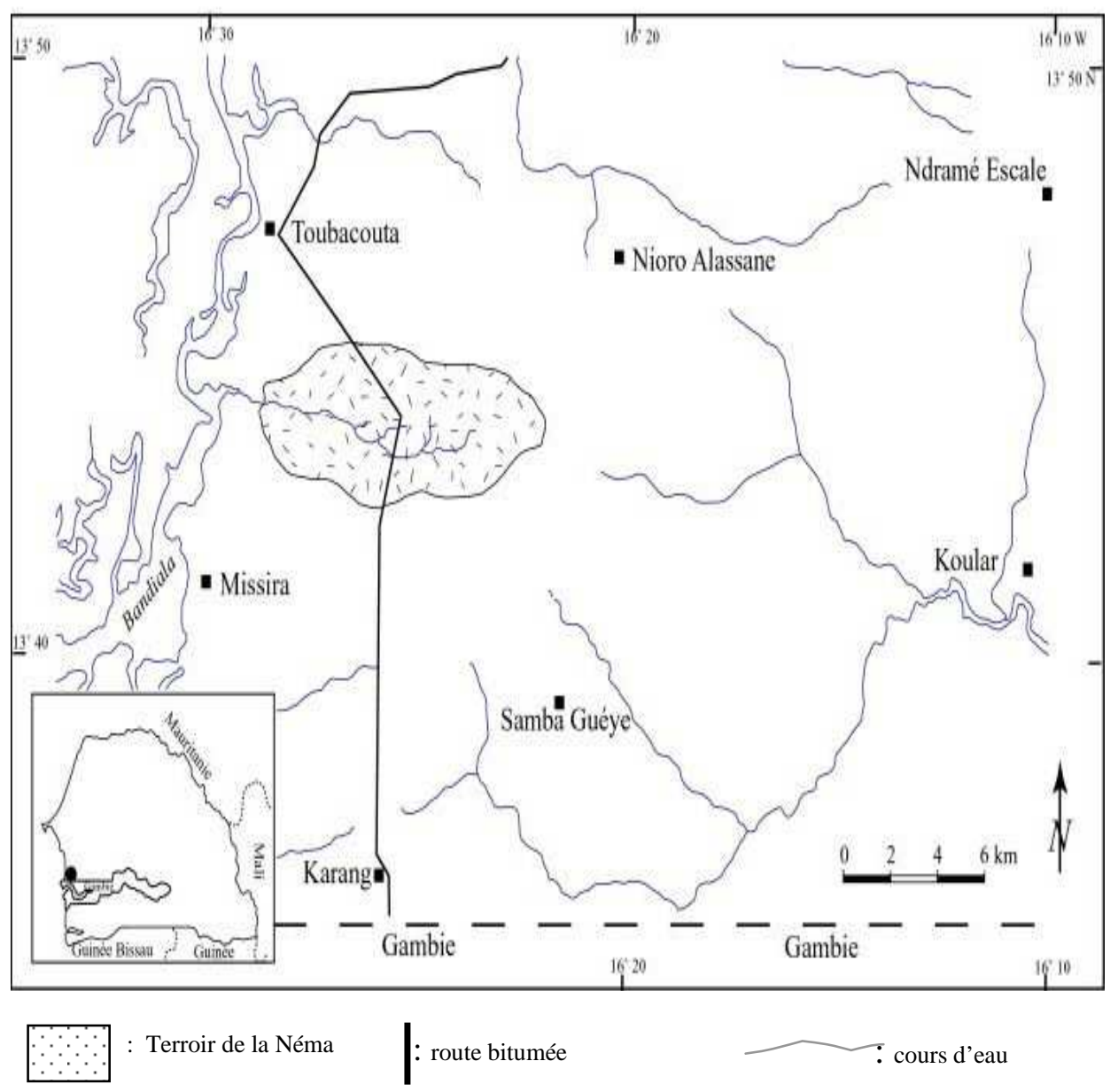

Figure 1 : Carte de situation du terroir de la Néma.

La production moyenne par exploitation est nettement plus élevée dans les grandes exploitations, en raison certainement de la taille des exploitations et de l'importance des facteurs de production. En effet, dans ce groupe, il y a les animaux de trait (bovins, équins et asins) qui sont utilisés dans les différentes activités agricoles. Ainsi la surface emblavée est importante (5,75 ha) mais leur effectif est peu important de sorte que globalement la production du groupe est inférieure à celle des exploitations moyennes.

Toujours dans les grandes exploitations, les marges d'amélioration des rendements sont importantes à condition de disposer de moyens de transport; la charrette à traction animale est tout à fait indiquée, voire indispensable pour l'utilisation de la fumure organique (Azouma et al., 2007 ; Vall et Blanchard, 2009). Ceci se traduit parfaitement sur la production moyenne de fane d'arachide par exploitation. En effet cette production plus élevée pour le groupe B1, puis les groupes A, B2 et enfin B3 est parfaitement corrélée à la possession d'animaux de trait (bovins) pour les travaux champêtres, aux superficies emblavées et à la quantité de fumier épandue. Les exploitants du groupe B1 disposent tous de bovins. 


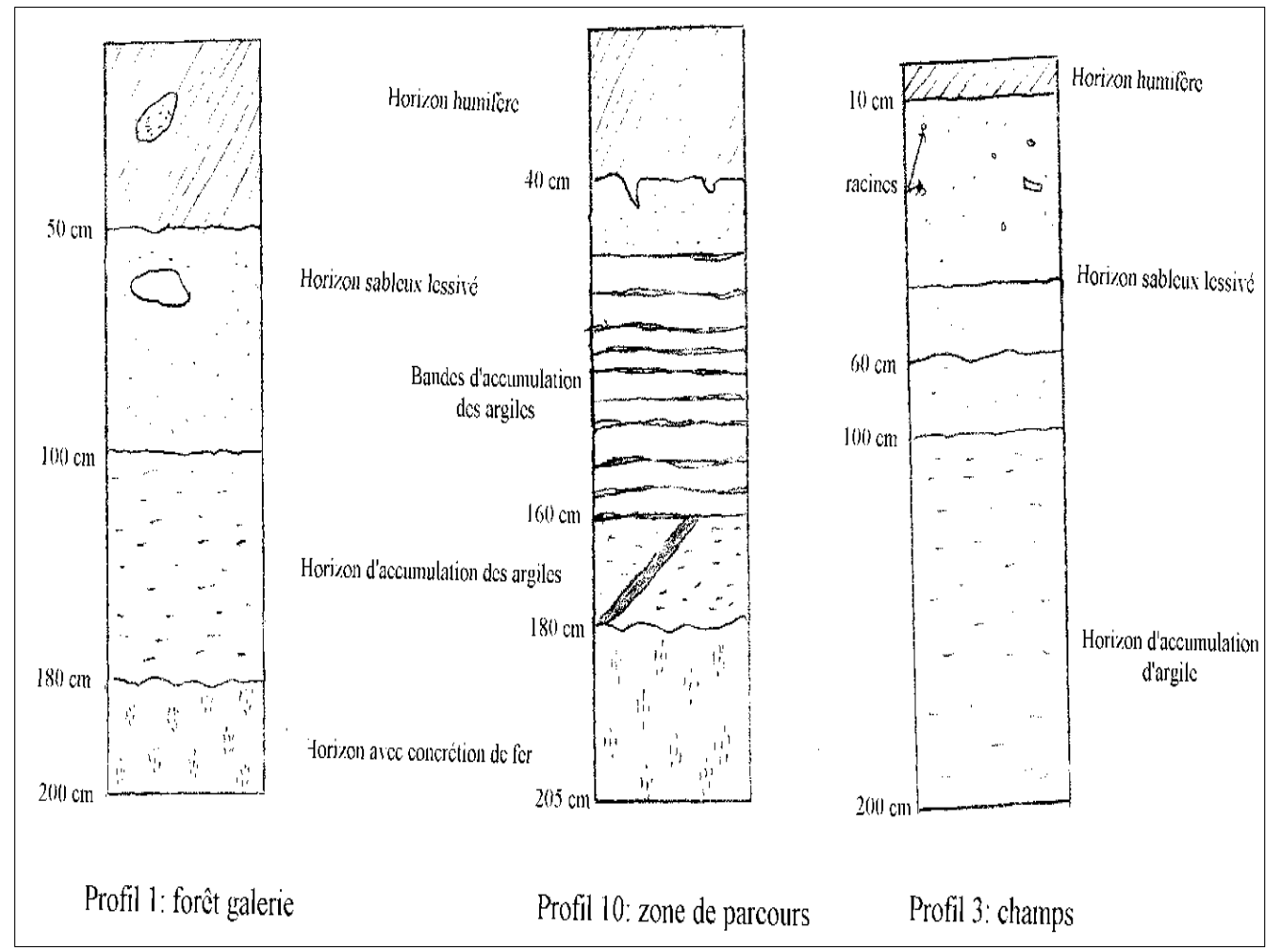

Figure 2 : Exemples de profils pédologiques dans la forêt galerie, la zone de parcours et dans les champs.

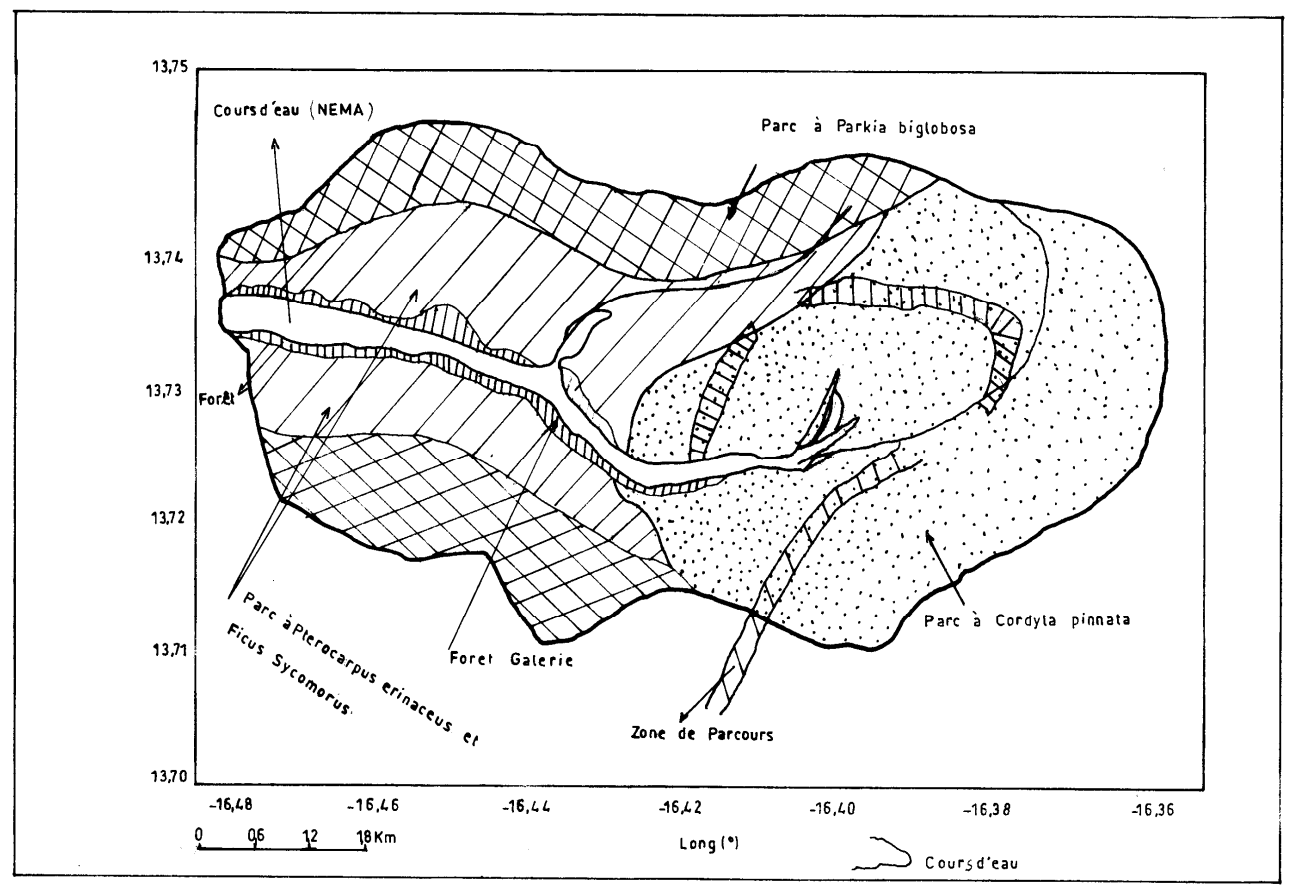

Figure 3 : Parcs agroforestiers du terroir de la Néma (Coly et al., 2005). 


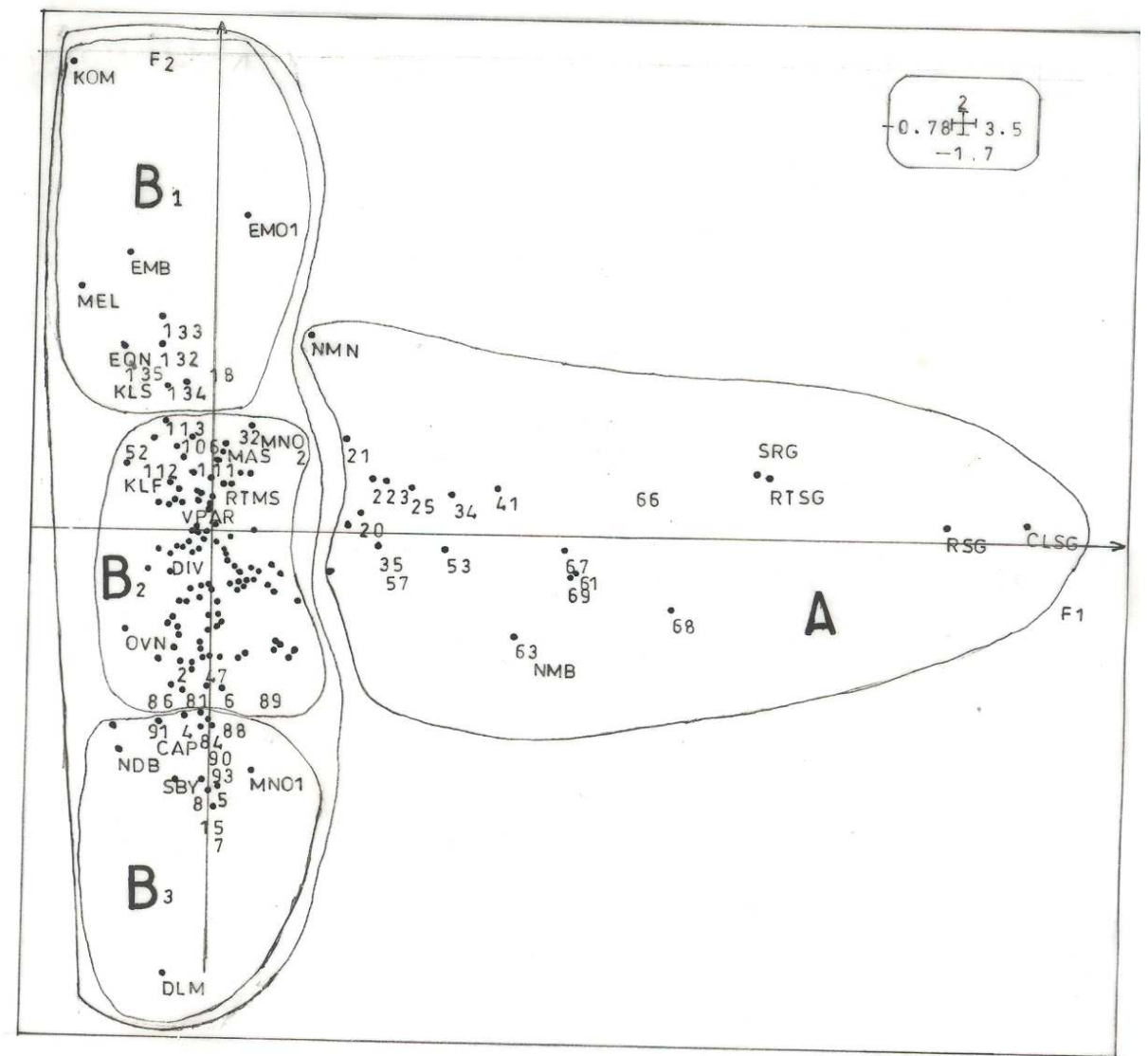

Figure 4 : Identification des groupes d'exploitations par AFC de 135 exploitations x 9 variables.

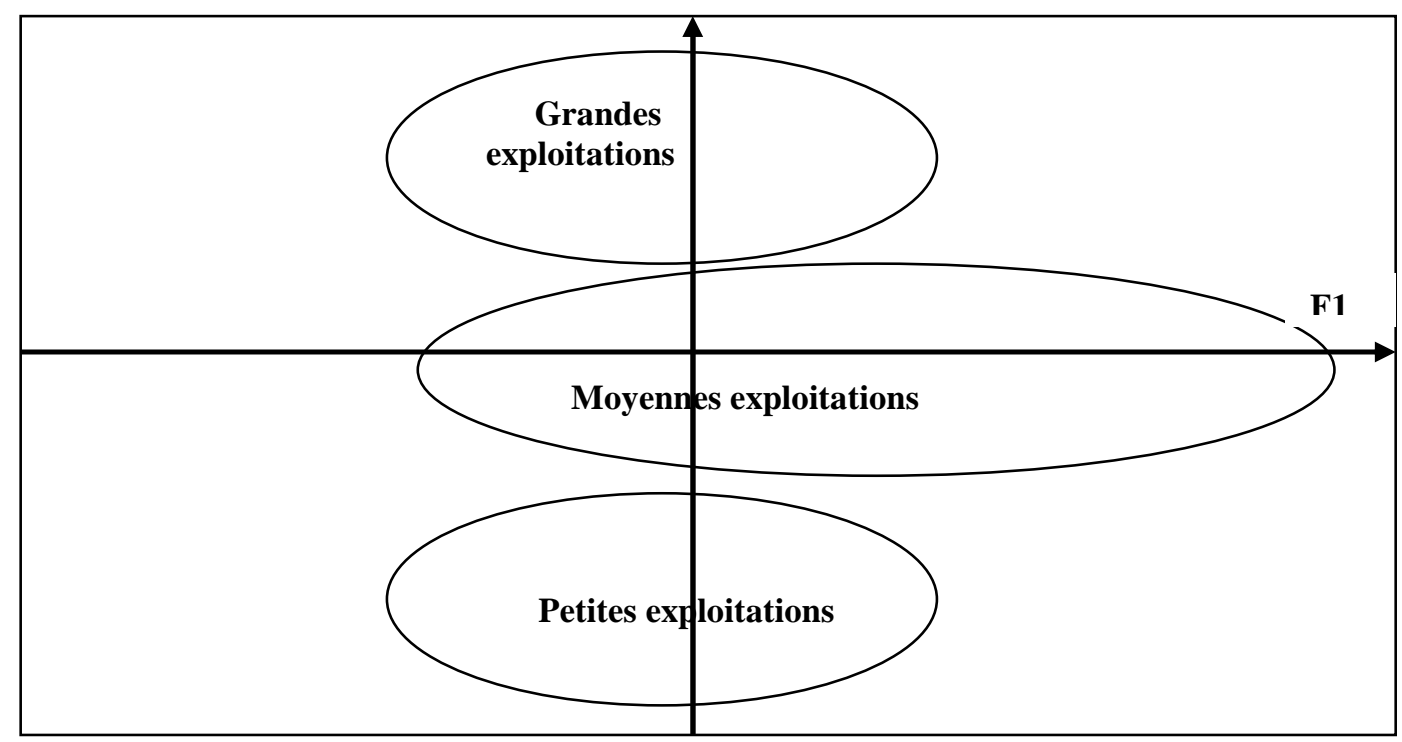

Figure 5: Discrimination des groupes d'exploitations agricoles en fonction de la taille dans le bassin de la Néma (Sénégal, West-Africa). 
Plus la taille des exploitations est petite, moins elle est performante. Les petites exploitations ne comptent que 5 personnes en moyenne ou 3 actifs agricoles en moyenne. Dans ce groupe, peu d'exploitants (36\%) disposent d'animaux de trait, la surface emblavée est petite (1,6 ha). L'usage du fumier y est très peu répandu $(0,36$ charrettées /an). Ces résultats corroborent ceux de Djondang et Gafsi (2003) selon lesquels la disponibilité en traction animale est un critère d'appréciation de la performance des exploitations agricoles.

La production moyenne par exploitation apparaît ainsi directement liée aux facteurs de production (animaux de trait, superficies emblavées...). Ces derniers dépendent aussi de la taille de l'exploitation. Par contre, la production globale dépend surtout du nombre d'exploitations par groupe et dans une moindre mesure de la taille des exploitations et de la disponibilité des facteurs de production.

\section{Les utilisations des sous-produits agricoles}

Les résidus de récolte produits sont soit ramassés, soit laissés sur place dans les champs.

Les fanes d'arachide constituent l'aliment essentiel des animaux de trait; elles sont entièrement ramassées. Une faible proportion est vendue. Le revenu de la vente permet d'acquérir du matériel agricole ou des petits ruminants. La vente n'intervient aussi que lorsque le chef d'exploitation ne possède pas d'animaux de trait ou en cas d'excédent. Dans ce dernier cas, elle intervient tard dans la saison sèche. Nous n'avons pas aussi de l'entraide dans la gestion des fanes d'arachide. La partie non vendue, représentant la plus grande quantité, est destinée à l'alimentation du bétail notamment les animaux de trait (bovins, asins et équins) gardés en stabulation. Les restes de cette partie destinée à l'alimentation du bétail, mélangés aux fèces et aux urines des animaux forment le fumier. Ce fumier sera collecté et transporté vers les champs pour relever leur fertilité.
La paille de mil est utilisée comme matériau de construction des enclos ou des clôtures des concessions. Une faible proportion est donc ramassée. La plus grande partie est laissée dans les champs pour servir de fourrages aux animaux en élevage extensif.

Vers la fin de la saison sèche, le reste de la paille est mis en tas pour être brûlé pendant la préparation des champs. C'est une autre forme de restitution de certains éléments minéraux ( $\mathrm{P}$ et $\mathrm{K}$ ) dans des proportions assez importantes (90\%). Ce procédé entraîne certainement un gaspillage de matière organique (Greenland,1977) qui en combinaison avec les intrants extérieurs (engrais minéral) permet d'augmenter les rendements (Pichot et Burdin, 1974). Dans ces conditions en effet, l'azote est entièrement perdu.

Le maintien du statut organique des sols est aussi lié à la disponibilité en fumure organique de l'exploitation (Djenontin et al., 2003 ; Annabi et al., 2009). En effet, le taux de matière organique du sol reste stable si l'on apporte 5 tonnes/ha de ce type de fumure tous les 2 ans (Sedogo, 1981) ou 7,5 tonnes à l'ha tous les 3 ans (Pol, 1991 ; Ridder et Keulen, 1990). L'enfouissement de la paille des céréales permet de conserver la matière organique et donc de maintenir la fertilité du sol. Cette pratique est inexistante dans le terroir. La paille est intégralement brûlée. Cette situation contribue ainsi à la dégradation du sol.

Il existe d'autres alternatives techniques pour entretenir le statut organique du sol comme le recyclage de la biomasse végétale, l'association arbres et cultures ou les plantes de couverture (Dugué, 1989).

La paille de maïs n'est pas ramassée, par conséquent elle est amassée en tas et brûlée sur place lors de la préparation des champs. Cela constitue une énorme perte d'éléments nutritifs et de matière organique. La conséquence est la baisse de la fertilité du sol. Les chaumes de maïs ne sont pas adaptés pour la confection de clôture.

La paille de sorgho comme celle de mil est utilisée pour les clôtures et les enclos 
surtout dans le village de Néma Ba (groupe d'exploitations A). Une partie importante est laissée dans les champs pour être brûlée.

Dans le terroir de la Néma, il existe de réelles disponibilités en paille de céréales alors que toute la fane d'arachide produite est ramassée pour l'alimentation animale et la vente. Les animaux de trait sont ainsi alimentés pendant toute l'année avec de la fane mais aussi et surtout en saison sèche. Il arrive parfois des ruptures de stock de fane d'arachide vers la fin de la saison sèche ; c'est la période de soudure. C'est à cette occasion que les pailles sont utilisées dans l'alimentation $\mathrm{du}$ bétail. Ces résultats corroborent ceux obtenus par Allard et al. (1983).

Il apparaît une forte corrélation entre la quantité de fumure animale répandue dans les champs et l'effectif d'exploitants possédant des bovins.

La fumure animale est utilisée prioritairement dans les champs destinés aux cultures vivrières, particulièrement le mil. Quant à l'engrais chimique, il est largement adopté (plus de $82 \%$ des exploitants) pour les cultures de rente, l'arachide (Ndao, 2000). L'engrais utilisé le plus couramment dans les champs d'arachide est le NPK 62010.

Dans le terroir, comme dans d'autres savanes de l'Afrique soudanienne et sahélosoudanienne, il semble émerger une économie agropastorale caractérisée par la diversification des sources d'alimentation du bétail par l'utilisation des sous produits agricoles, la fertilisation organique et la traction animale.

\section{Conclusion}

Les caractéristiques des exploitations ont permis d'en distinguer trois groupes principaux ; ce sont les grandes exploitations, les exploitations moyennes et les petites exploitations.

La production moyenne de fane d'arachide par exploitation apparaît toujours plus élevée dans le groupe des grandes exploitations et plus faibles dans les petites exploitations pour les deux campagnes agricoles d'observation.
Toutefois, la production totale est plus importante dans le groupe des exploitations moyennes; elle reste toujours plus faible dans le groupe des petites exploitations.

Dans le terroir de la Néma, la gestion des résidus de récolte dépend des formes d'utilisation. La fane d'arachide est entièrement ramassée et utilisée presqu'exclusivement dans l'alimentation du bétail. Une faible proportion est destinée à la vente. La paille de mil est ramassée dans $80 \%$ des exploitations pour la confection des clôtures et des parcs pour les animaux de trait. Une importante partie est laissée dans les champs, comme les pailles de maïs ou de sorgho pour être brûlée lors de la préparation des champs. Cette pratique permet la restitution de certains éléments minéraux au sol mais entraîne aussi une perte énorme de matière organique et d'azote pourtant très déterminants dans la fertilité des champs.

La partie de la fane utilisée dans l'alimentation des animaux de trait a permis d'obtenir du fumier à partir des restes, des fèces et des urines des animaux qui sont ramassés et mis en tas dans la concession de l'exploitant. Le facteur de conversion de la fane distribuée comme aliment en fumier est très faible.

Pour améliorer la fertilité des champs et donc la production dans ces différents groupes d'exploitations, un certains nombre de mesures sont nécessaires comme :

- l'enfouissement des pailles de mil, de sorgho et de maïs généralement brûlées; ce qui permettrait de conserver en plus du phosphore $(\mathrm{P})$ et du potassium $(\mathrm{k})$, l'azote $(\mathrm{N})$ et la matière organique ;

- l'apport de litière dans les enclos des animaux en vue d'améliorer la qualité du fumier par une meilleure rétention de l'azote contenu dans les fèces et les urines voué à la volatilisation mais aussi par une amélioration du statut organique du fumier;

- l'augmentation des quantités de fumier apportées au champ généralement faible dans les exploitations agricoles.

La connaissance des caractéristiques de chaque groupe d'exploitations ainsi défini 
constitue un gage à la réussite de toute politique de gestion durable des ressources agricoles du terroir de la Néma. Cependant il semble opportun d'apprécier la rentabilité des différents modes de gestion des résidus de récolte et leur impact sur la fertilité du sol suivant les groupes d'exploitations.

\section{REFERENCES}

Akpo LE, Coly I, Sarr D, Ngom D, Ndao S. 2003. Modes d'occupation des terres et gestion des ressources forestières en zone soudannienne de l'Afrique de l'ouest: l'exemple du bassin versant de la Néma au Sénégal. Actes de colloque, Montpellier, 1-8.

Akpo LE, Coly I, Sarr D, Ngom D, Ndao S. 2002. Impact des modes d'utilisation des terres sur la diversité floristique dans le bassin de la Néma en zone soudanosahélienne (Sine-Saloum, Sénégal). In $5^{\text {ème }}$ Conférence interrégionale sur l'environnement et l'eau, Maïga $\mathrm{AH}$, Pereira LS, Musy A (éds). Actes de colloque, Ouagadougou, SOGIF, 306317.

Annabi A, Bahri H, Latiri K 2009. Statut organique et respiration microbienne des sols du nord de la Tunisie. Biotechnol. Agron. Soc. Environ. 13 (3) 401-408

Azouma OY, Giroux F, Varchen D. 2007. Conception d'un épandeur de fumures organiques pour les exploitations à traction animale d'Afrique. Tropicultura, 25(2) : 75-81.

Bacco MN, Djenontin MJ, Amidou M. 2003. Gestion de la fertilité des sols dans le nord Benin et incidences économiques pour les exploitations agricoles. Savanes africaines des espaces en mutations, des acteurs face à de nouveaux défis. Actes de colloque, mai 2002, Garoua, Cameroun.

Coly I, Akpo LE, Dacosta H, Diome F, Malou R. 2001. Caractérisation agro-écologique du terroir de la Néma au Sine Saloum (Sénégal) : les systèmes d'utilisation des terres et diversité des peuplements ligneux. Journal des Sciences, 1(2): 9-18.
Coly I, Akpo LE, Sarr D, Malou R, Dacosta H, Diom F. 2005. Caractérisation agroécologique du terroir de Néma en zone soudano-sahélienne au Sénégal : typologie des parcs agroforestiers. Agronomie Africaine, 17(1): 53-62.

Coly I. 1999. Caractérisation des ressources ligneuses du terroir de la Néma dans le Niombato (Saloum) au Sénégal. DEA FST/UCAD, Dakar, 38 p.

Djenontin JA, Amidou M, Bacco M, Wennink B. 2003. Valorisation des résidus de récolte dans l'exploitation agricole au nord du Benin: production de fumier et enfouissement des résidus de récolte pour la gestion de la fertlité des sols. Organisation spatiale et gestion des ressources et des territoires ruraux. Actes de colloque international 25-27 fevrier 2003 Montpellier, France, 11 p.

Djondang K, Gafsi M. 2003. Caractérisation des exploitations agricoles en zone soudanienne du Tchad. Savanes africaines : des espaces en mutation, des acteurs face à de nouveaux défis. Actes du colloque, mai 2002, Garoua Cameroun, Prasac, N'Djamena, Tchadcirad, Montpellier, France, 9 p.

Dugue P. 1989. La culture attelée en zone sahélo-soudanienne : solution viable pour le développement agricole ou utopie agronome? Le cas du Yatenga au Burkina Faso. Cab. Rech.-Dév., 21: 4559.

Greenland DJ. 1977. Organic recycling in agriculture: Some research needs in Organic recycling in Africa. Papers presented at the FAO/SIDA Workshop on the use of organic materials as fertilizers in Africa held in Buea, Cameroon, 5-14.

Hauhouot C. 2004. Les pressions anthropiques sur milieux naturels du sud-ivoirien. GeoEco-Trop., 28(1-2): 69-82.

Kaasschieter GA, Coulibaly Y. 1995. Rentabilité de l'utilisation de fane de niébé (Vigna unguiculata) comme supplément avec la paille de mil (Pennisetum typhoides) par des taurillons. Rapport Production Soudano-Sahélienne 
$\mathrm{N}^{\circ}$ 7. Exploitation optimale des éléments nutritifs en élevage, $29 \mathrm{p}$.

Lienou G. 1995. Relations écoulements de surface-écoulements souterrains dans le bassin versant de la Néma (Sine-Saloum, Sénégal). Thèse de DEA, Université Cheikh Anta Diop de Dakar.

Mendy A. 2000. Etude hydrologique du bassin versant de la Néma et de la mise en valeur de ses bas-fonds. Mém. Maîtrise de Géographie, FLSH, UCAD, Dakar.

Ndao S. 2001. Pratiques agricoles et gestion de l'arbre dans les parcs agroforestiers de la Néma en zone soudano-sahélienne (Sine-Saloum, Sénégal). DESSS, CRESA, Niamey, $70 \mathrm{p}$.

Ngom D. 2000. Importance de l'arbre dans les systèmes de production traditionnels du terroir de la Néma dans le Niombato (Saloum, Sénégal). DESS, CRESA, Niamey, $72 \mathrm{p}$.

Pichot J, Burdin S. 1974. L'enfouissement des pailles de mil Pennisetum dans les sols.

Pol F. 1991. L'épuisement des terres, une source de revenus pour les paysans du Mali -Sud. In Savanes d'Afrique, Terres
Fertiles? Ed. Focal Coop, Ministère de la Coopération et du Développement: Paris ; 403-418.

Razafindrakoto MA. 2007. Stratégie améliorée pour l'accroissement de la fertilité du sol et de sa résistance à l'érosion (régions centre des hautsplateaux de Madagascar). Actes de JSIRAUF, Hanoi, 6 p.

Ridder N, De Keulen H. 1990. Some aspects of the role of organic matter in sustainable intensified arable farming systems in the West-Africa semi-arid tropics. Fertilizer Research, 26: 299-310.

Sédogo MP. 1981. Contribution à la valorisation des résidus culturaux en sol ferrugineux et sous climat tropical semiaride (matière organique du sol et nutrition azotée des cultures). Thèse Docteur Ingénieur INPL, Nancy, 198 p.

Vall E, Blanchard M. 2009. Composter les tiges de cotonnier au champ pour accroitre la production agricole en respectant l'environnement et en réduisant les charges. Projet Fertipartenaires-CIRAD, 3p. 